\title{
Einige Bemerkungen über das Arginin. \\ Von
}

E. Schulze.

(Der Redaction zugegangen am 18. März 1900.)

Ob die Arginin-Präparate verschiedener Herkunft gleiche Eigenschaften besitzen oder nicht, das ist eine Frage, die der Prüfung bedarf. Mit dieser Prüfung hat W. Gulewitsch ${ }^{1}$ ) sich beschäftigt; er verglich "thierisches Arginin", dargestellt aus Heringssperma, mit dem von mir und meinen Mitarbeitern untersuchten "pflanzlichen Arginin" und glaubte dabei eine sehr grosse Verschiedenheit der beiden Substanzen im specifischen Drehungsvermögen gefunden zu haben. Doch beruhte letzteres auf einem Missverständniss, wie aus dem Nachtrag $\mathrm{zu}$ ersehen ist, den W. Gulewitsch${ }^{2}$ ) seiner Abhandlung folgen liess; denn die in der bezüglichen Mittheilung von E. Steiger und mir angegebenen Zahlen bedeuten nicht, wie Gulewitsch glaubte, Kreisgrade, ${ }^{3}$ ) sondern Grade der Scala

1) Diese Zeitschrift, Bd. XXVII, S. 178.

2) Ebendaselbst, S. 368 .

8) Dass unsere Angaben missverstanden worden sind, hat mich sehr überrascht. Nach meiner Ansicht ist doch leicht zu sehen, dass E. Steiger und ich in den Sätzen: «Eine wässrige Lösung, welche in $20 \mathrm{ccm} .2,0 \mathrm{~g}$ Argininnitrat enthielt, drehte im Soleil-Ventzke'schen Polarisationsapparat im $200 \mathrm{~mm}$.-Rohr $5,75^{\circ}$ nach rechts und «Eine wässrige Lösung, welche in $50 \mathrm{ccm} .4,0 \mathrm{~g}$ salzsaures Arginin enthielt, drehte im Soleil-Ventzke'schen Polarisationsapparat im $200 \mathrm{~mm}$.-Rohr $5,3^{\circ}$ nach rechts die bei der Untersuchung jener Salze im genannten Apparat unmittelbar erhaltenen Ergebnisse mittheilen. Es würde doch seltsam sein und nicht dem Usus entsprechen, wenn wir die im SoleilVentzke'schen Apparat gemachten Beobachtungen nicht in Graden der diesem Apparat eigenthümlichen Scala, sondern in Kreisgraden an- 
des Soleil-Ventzke'schen Apparates. Aus diesen Zahlen berechnen sich für Argininnitrat $[\varkappa] D=+9,95$, für Argininchlorid $[a] \mathrm{D}=+11,45$ - Werthe, welche von den von Gulewitsch gefundenen Zahlen $([\alpha] \mathrm{D}=+9,31$ für Argininnitrat und $=+10,70$ für Argininchlorid) nur wenig abweichen. Ich kann hinzusetzen, dass die Differenzen innerhalb der Fehlergrenze unserer Bestimmungen liegen; denn der damals von uns verwendete Apparat gestattete nicht, bei Ermittelung des Drehungsvermögens der zur Untersuchung gelangenden Substanzen eine grössere Genauigkeit zu erreichen.

Die bei der Untersuchung im Polarisationsapparat erhaltenen Resultate geben also, wie auch Gulewitsch meint, keine Veranlassung, das thierische Arginin für verschieden vom pflanzlichen zu erklären; ebensowenig führten zu einer solchen Annahme die übrigen Resultate, zu denen Gulewitsch in seiner Arbeit gelangte. Denn die Beobachtungen, welche dieser Forscher über die Eigenschaften und über die Zusammensetzung des Argininchlorids, des Argininnitrats und des Argininkupfernitrats machte, sind fast in allen Stücken Bestätigungen der bezüglichen Angaben von E. Steiger und mir; nur in wenigen Punkten sind kleine Abweichungen hervorgetreten. Auf diese Abweichungen will ich im Folgenden näher eingehen.

Was zunächst das Argininchlorid betrifft, so hatte Gulewitsch ein Präparat dieses Salzes unter Händen, in welchem ein Molekül Krystallwasser gefunden wurde, ${ }^{1}$ ) während das von E. Steiger und mir untersuchte Präparat wasserfrei

gegeben hätten, ohne in letzterem Falle zu erwähnen, dass die Kreisgrade durch Berechnung aus den an der Scala des genannten Apparates abglesenen Werthen erhalten wurden. Uebrigens hätte schon ein Blick in irgend eine andere meiner Abhandlungen, in welcher Angaben über das specifische Drehungsvermögen irgend welcher Substanzen sich finden, zeigen können, wie die obigen Sätze zu verstehen sind; denn ich habe dort stets die Ergebnisse der polarimetrischen Bestimmungen in analoger Weise mitgetheilt, jedoch unter Hinzufügung der für $[\alpha] D$ berechneten Werthe.

1) Das Gleiche gilt für ein von S. G. Hedin untersuchtes Präparat des gleichen Salzes; dieses Präparat verlor das Krystallwasser schon bei $100^{\circ}$. 
war. Doch waren diese beiden Präparate nicht genau in der gleichen Weise dargestellt; das unsrige war nämlich aus Wasser, das von Gulewitsch untersuchte dagegen aus verdünntem Weingeist umkrystallisirt worden. Da von E. Steiger und mir mehrere Analysen des Argininchlorids ausgeführt worden sind, so war es sehr unwahrscheinlich, dass unsere Angabe über das Fehlen von Krystallwasser in diesem Salz auf einem Irrthum beruhte; um indessen völlig sicher $\mathrm{zu}$ sein, habe ich ein von früher her in meinen Händen befindliches Präparat des Chlorids noch einmal untersucht. Dabei ergab sich, dass das Salz beim Erhitzen auf $130-135^{\circ}$ im Luftbade nicht an Gewicht verlor.

Das Argininchlorid vermag also sowohl mit als ohne Krystallwasser zu krystallisiren.

Ferner erhielt Gulewitsch bei Bestimmung des Krystallwassers im Argininkupfernitrat Zahlen, welche der Formel $\left(\mathrm{C}_{6} \mathrm{H}_{14} \mathrm{~N}_{4} \mathrm{O}_{2}\right)_{2} \mathrm{Cu}\left(\mathrm{NO}_{3}\right)_{2}+3^{1 / 2} \mathrm{H}_{2} \mathrm{O}$ entsprechen, während sowohl von $\mathrm{E}$. Steiger und mir als von S. G. Hedin für die genannte Verbindung eine Formel mit drei Molekülen Krystallwasser angenommen worden ist. Ich bemerke dazu, dass von meinen Mitarbeitern und mir mehr als 10 Präparate von Argininkupfernitrat verschiedener Herkunft untersucht worden sind und dass die dabei erhaltenen Resultate sämmtlich zu einer Formel mit 3 Molekülen Krystallwasser führen. Der letzteren Formel entsprechen auch die bei Bestimmung des Kupfergehalts jener Präparate gefundenen Zahlen besser, als einer Formel mit $3^{1 / 2}$ Molekülen Krystallwasser. Allerdings übersteigen die bei der Krystallwasserbestimmung von uns erhaltenen Resultate zum Theil nicht ganz unbeträchtlich die Werthe, welche aus der von uns gegebenen Formel sich berechnen, wie schon aus der ersten, von E. Steiger und mir über das Arginin gemachten Mittheilung $\mathrm{zu}$ ersehen ist ${ }^{1}$ ) doch ist das Plus nicht so gross, dass es zur Aufstellung einer Formel mit $3^{1 / 2}$ Molekülen Krystallwasser führen könnte.

1) Statt der berechneten $9,16 \%$ fanden wir $9,37-9,54 \%$ Krystallwasser. 
Die kleine Abweichung, welche in Bezug auf diesen Punkt zwischen unseren Angaben und den Angaben von Gulewitsch hervorgetreten ist, würde sich erklären, wenn man annimmt, dass das Argininkupfernitrat schon beim Liegen an der Luft oder im Exsiccator über Chlorcalcium einen kleinen Theil seines Krystallwassers verlieren kann. Für die Identität des von Gulewitsch und des von uns untersuchten Argininkupfernitrats spricht übrigens noch die Thatsache, dass die über den Schmelzpunkt unserer Präparate gemachten Beobachtungen der von Gulewitsch gemachten Angabe $\left(112-114^{\circ}\right)$ entsprechen.

Wie aus den im Vorigen gemachten Mittheilungen zu ersehen ist, liegt kein Grund vor, eine Verschiedenheit des thierischen und des pflanzlichen Arginins anzunehmen. Man kann nun noch die Frage aufwerfen, ob das im pflanzlichen Stoffwechsel entstandene Arginin in seinen Eigenschaften vollständig mit dem beim Kochen der Eiweissstoffe mit Säuren sich bildenden Arginin übereinstimmt. In Beantwortung dieser Frage ist auf eine kleine Verschiedenheit aufmerksam zu machen, welche zwischen den Arginin-Präparaten, die jenen beiden Quellen entstammen, hervorgetreten ist. Aus den PflanzenSäften und -Extracten kann man, wie von mir an mehreren Objecten gezeigt worden ist, Arginin durch Mercurinitrat ausfällen ${ }^{1}$ ); auch das bei Zerlegung dieser Niederschläge resultirende Argininnitrat gibt in wässeriger Lösung mit Mercurinitrat eine Fällung. Isolirt man das Arginin aus den Pflanzenextracten mit Hülfe von Phosphorwolframsäure und führt das bei Zerlegung des bezüglichen Niederschlags erhaltene Arginin in das Nitrat über, so resultiren gleichfalls Präparate, die mit Mercurinitrat einen Niederschlag geben; sie behalten diese Eigenschaft auch, wenn man sie durch Ueberführung in die schwerlösliche Kupferverbindung einer Reinigung unterworfen hat. Das Nitrat des durch Spaltung von Eiweissstoffen mit Säuren erhaltenen Arginins gibt dagegen unter gleichen Umständen mit Mercurinitrat keine Fällung. Andere Verschieden-

1) Doch ist die Ausfällung keine vollständige. 
heiten zwischen den aus Eiweissstoffen und den aus Keimpflanzen dargestellten Argininpräparaten haben wir aber bis jetzt nicht auffinden können. Auch geht aus den von E. Winterstein und mir ausgeführten Versuchen hervor, dass Arginin, mag es nun der einen oder der anderen Quelle entstammen, beim Erhitzen mit Barytwasser Ornithin und Harnstoff als Zersetzungsprodukte liefert.

Man kann es wohl nicht für ganz unwahrscheinlich erklären, dass dem aus Keimpflanzen dargestellten Arginin seine Eigenschaft, durch Mercurinitrat gefällt zu werden, durch eine nur in sehr geringer Menge vorhandene Verunreinigung gegeben wird. Allerdings gaben die jener Quelle entstammenden Argininnitrat-Präparate auch dann, wenn sie möglichst gut gereinigt worden waren, noch Fällung mit Mercurinitrat; doch schienen die Niederschläge allmählich schwächer zu werden. Vielleicht gelingt es noch, eine Reinigungsmethode aufzufinden, durch welche man den Argininnitrat-Präparaten die Eigenschaft, durch Mercurinitrat gefällt zu werden, nehmen kann. Uebrigens wird die Darstellung von Arginin aus Pflanzenextracten durch sein Verhalten gegen Mercurinitrat in vielen Fällen erleichtert. Doch geht in den Mercurinitrat-Niederschlag nach den mit Lupinus-Keimpflanzen von uns gemachten Versuchen neben Arginin auch Histidin ein.

Im Hinblick auf die vor Kurzem erschienene Abhandlung Thompson's ${ }^{1}$ ) über die physiologische Wirksamkeit der Protamine und ihrer Spaltungsprodukte sei hier noch daran erinnert, dass auch nach Versuchen, welche früher schon von Schmiedeberg ausgeführt wurden, ${ }^{2}$ ) das Arginin im Thierkörper keine giftige Wirkung hervorbringt.

1) Diese Zeitschrift, Bd. XXIX, S. 1.

2) Berichte der d. chem. Gesellsch., Bd. XXIX, S. 355. 